Methods Continuous interscalene brachial plexus block for the shoulder or humerus surgery patients was assessed using 8 domestic databases including Korea Med and Ovid-MEDLINE, Ovid-EMBASE and Cochrane Library. Through a search strategy, a total of 348 works were identified and total of 21 works were included in the final assessment. Two reviewers screened all references independently, for assessing included articles quality and extracted data.

Results Side effects of the drug were reported to have occurred less or at similar level in the intervention group than the control group, although procedure related complications were reported to have occurred more often in the intervention group compared to the control group. The average pain score following the surgery was reported to be significantly lower or similar for the intervention group compared with the control group. The intervention group also had lower or similar level of quantity of additional analgesics used in comparison to the control group. In addition, the intervention group displayed either similar or higher level of satisfaction of patients on the pain control method.

Conclusion Continuous interscalene brachial plexus block was assessed to be a safe and effective technology when used for the purpose of reduction in pain in shoulder or humerus surgery patients since it was found to be relatively safe when compared with the existing procedures, and similar or more effective in terms of pain control and usage of additional analgesics in comparison to the existing pain control methods.

\section{P181 SNORING, GASPING AND ADAPTE!}

D Bruinvels. Netherlands Society of Occupational Medicine - Centre of Excellence, Utrecht, The Netherlands

\section{0:1136/bmjqs-2013-002293.195}

Background The Netherlands Society of Occupational Medicine (NVAB) has a tradition of guideline development since 1999. The development of a typical monodisciplinary guideline for occupational physicians takes two years. In order to save time and costs the NVAB initiated an ADAPTE process for a monodisciplinary guideline on Obstructive Sleep Apnea Syndrome (OSAS).

Context OSAS is a major problem in the working population and may attribute to a substantial proportion of work related problems associated with fatigue. Examples are work accidents, work errors and productivity loss. Although a Dutch multidisciplinary guideline on OSAS exists, only a minority of the Dutch occupational physicians actually uses this guideline. To facilitate the implementation of the guideline, the NVAB provided a summary, a PowerPoint presentation, and medical case studies for occupational physicians. However, the guideline was still not implemented in clinical practice. Therefore the NVAB took the initiative to develop a monodisciplinary practice guideline for occupational physicians.

Description The ADAPTE process was used to develop a monodisciplinary practice guideline for occupational physicians based on a Dutch multidisciplinary guideline developed by $\mathrm{CBO}$ in 2009. The aim was to develop a monodisciplinary guideline in one year. In the end it took almost 3 years to adapt the guideline.

Lessons ADAPTE is a practical tool to adapt multidisciplinary guidelines into monodisciplinary practice guidelines. However, the use of the ADAPTE process does not guarantee time savings and reduction of development costs.

\section{P184 INCLUDING WORK PARTICIPATION IN DUTCH MULTIDISCIPLINAIRY GUIDELINES - AN OVERVIEW}

D Bruinvels. Netherlands Society of Occupational Medicine - Centre of Excellence, Utrecht, The Netherlands

\section{0:1136/bmjqs-2013-002293.196}

Background In 2006 an innovative programme for the development of multidisciplinary guidelines (KKCZ) was started in The Netherlands. The KKCZ programme aimed to develop multidisciplinary guidelines, to facilitate collaboration between professional groups, to enhance patient participation, and to avoid duplication of efforts on a national level. Context One of the innovative aspects of the KKCZ-programme was to stimulate guideline developers to include (work) participation in the guidelines. Therefore The Netherlands Society of Occupational Medicine (NVAB) has developed a "Blueprint Participation in Guidelines" to facilitate guideline developers to include work participation in KKCZ-guidelines.

Description The KKCZ-programme has led to 53 multidisciplinary guidelines. In an evaluation study the contents of eight KKCZ-guidelines and two other multidisciplinary guidelines was assessed. This was accomplished by first developing a list of process indicators describing essential steps of the Blueprint. Second, the use of the Blueprint was evaluated using this list. All eight KKCZ-guidelines were developed using the Blueprint. Because process indicators do not give information on the actual contents of a guideline, a web based survey was held among Dutch occupational physicians. 253 occupational physicians reported on the inclusion of work participation in the guidelines. The majority found that work participation was included in these guidelines. However, they also found that many of the key recommendations in the guidelines were difficult to implement.

Lessons The use of a Blueprint for guideline developers has stimulated the inclusion of work participation in multidisciplinary guidelines. However, the use of this Blueprint is no guarantee that key recommendations on work participation are implemented in daily practice.

\section{P187 DEVELOPING A FRAMEWORK AND CRITERIA TO SORT, SELECT AND REDUCE MEASUREMENT INSTRUMENTS FOR DAILY PRACTICE IN PHYSICAL THERAPY}

${ }^{1} G$ Meerhoff, , ${ }^{2} S$ Beurskens, ${ }^{2} R$ Swinkels, ${ }^{4} \mathrm{P}$ van der Wees, ${ }^{3} \mathrm{~V}$ de Graaf-Peters. ${ }^{1}$ Royal Dutch Society for Physical Therapy (KNGF), Amersfoort, The Netherlands; 'Zuyd University of Applied Sciences, Heerlen, The Netherlands; ${ }^{3}$ Hanze University of Applied Sciences, Groningen, The Netherlands; ${ }^{4}$ Scientific Institute for Quality of Healthcare (IQ), Nijmegen, The Netherlands

\section{0:1136/bmjqs-2013-002293.197}

Objectives The amount and diversity of measurement instruments in the Dutch physiotherapy guidelines is too extensive: in total 111 instruments. Many of these instruments are too long and concern identical domains and concepts. The aim of this bottom-up project is to develop a framework and criteria that will guide the structure, selection and reduction of instruments for application in daily practice.

Methods The project consist of the next steps: 1. Defining the aim of the framework and review the (inter)national literature; 2. Developing a concept framework; 3 . An inventory of domains and overlap of instruments in the Dutch guidelines; 4. An inventory made by professionals of domains and concepts relevant for musculoskeletal functioning in physiotherapy and instruments used; 5. Consensus meetings with representatives of 